\section{Legless — but still the way forward}

The more complex a pathway, the more the output can be fine-tuned by incoming signals. Unfortunately, this also means that there are more opportunities for things to go wrong, as highlighted by the results of three independent studies led by He, Bienz and Basler.

The Wingless (Wg) — or mammalian Wnt — pathway transduces signals from the cell surface to the nucleus by preventing the glycogen synthase kinase 3 (GSK3)-mediated phosphorylation of Armadillo/ $\beta$-catenin. When phosphorylated, Armadillo/ $\beta$-catenin gets degraded. However, in the absence of phosphorylation - that is, in response to Wg or $\mathrm{Wnt}$ - Armadillo/ $\beta$-catenin shuttles to the nucleus to regulate transcription by the T-cell factor, TCF.

Beginning upstream in the Wnt pathway, $\mathrm{He}$ and colleagues found that four key amino-terminal $\beta$-catenin residues - Ser33, Ser37, Thr41 and Ser45 - must be phosphorylated in a carboxy- to amino-terminal direction for $\beta$-catenin to be degraded.

These four residues conform to a consensus GSK3 phosphorylation site, but does GSK3 phosphorylate them all? The authors showed that a separate 'priming' kinase was required to phosphorylate Ser45 before GSK3 could phosphorylate Thr41, Ser37 and Ser33. This 'Ser45 kinase' was identified as casein kinase I $\alpha(\mathrm{CKI} \alpha)$. Depletion of CKI $\alpha$ - but not its close relative $\mathrm{CKI} \varepsilon$ - by RNA-mediated interference caused $\beta$-catenin to accumulate, which indicates that CKI $\alpha$ is required for Ser 45 phosphorylation, the subsequent phosphorylation by GSK3 of Thr41, Ser37 and Ser33 and, ultimately, the degradation of $\beta$-catenin. Perhaps it is not surprising, then, that mutations at Ser33, Ser37, Thr41 or Ser45 are associated with colorectal cancer and that $\mathrm{CKI} \alpha$ is a candidate tumour suppressor.

Two more genes - legless and pygopus - that function in the Wg/Wnt pathway were identified by Basler's group using genetic screening. Pygopus was also identified by Bienz's group. As mutations in either of these genes suppressed the phenotype induced by overexpression of $\mathrm{Wg}$, this indicated that these proteins transduce, rather than produce, the Wg signal. Further genetic analysis showed that Legless and Pygopus function at the bottom of the Wg pathway, downstream of Armadillo/ $\beta$-catenin. Legless and Pygopus bind to one another, but Legless was also shown to bind Armadillo/ $\beta$ catenin. So Pygopus binds to Legless, Legless binds to Armadillo/ $\beta$-catenin, and Armadillo/ $\beta$-catenin signalling cannot occur without Legless and Pygopus. What, then, do these two proteins do?

Basler's group showed that Armadillo/ $\beta$-catenin can bind Legless and TCF simultaneously, hinting that Legless and Pygopus might affect Armadillo/ $\beta$-catenin-mediated transcription. As Pygopus enhanced TCFreporter-gene transcription in a tissue-culture assay, this indicates that the principal role of Legless could be to recruit Pygopus to $\beta$-catenin in the nucleus, where it influences gene transcription. How it does this is so far unclear. But at least we're moving in the right direction.

Katrin Bussell

\section{(2) References and links}

ORIGINAL RESEARCH PAPERS Liu, C. et al. Control of

$\beta$-catenin phosphorylation/degradation by a dual-kinase mechanism. Cell 108, 837-847 (2002) | Kramps, T. et

al. Wnt/Wingless signaling requires BCL9/Leglessmediated recruitment of Pygopus to the nuclear $\beta$-catenin-TCF complex. Cell 109, 47-60 (2002) Thompson, B. et al. A new

nuclear

component

Wnt signalling

pathway. Nature

Cell Biol. 4 ,

$367-373(2002)$

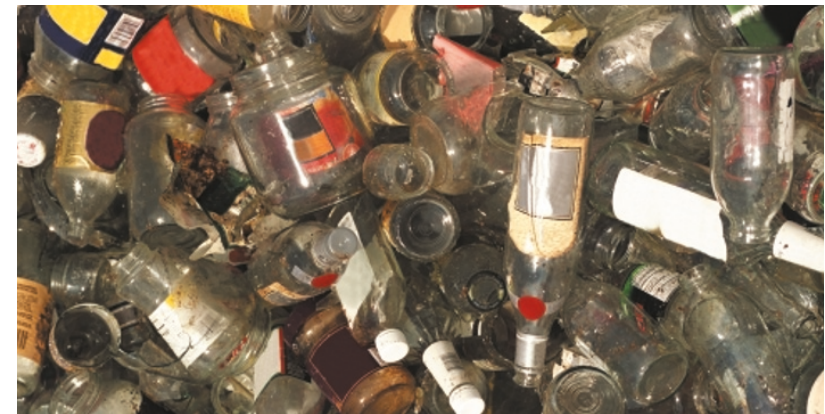

ENDOCYTOSIS

\section{A new recycling route}

Many cell-surface proteins are anchored to the membrane by glycosylphosphatidylinositol (GPI), and it has remained unclear how these GPI-anchored proteins (GPI-APs) are internalized by cells. But now, in Developmental Cell, Mayor and colleagues describe a new endocytic pathway that delivers these proteins to recycling endosomes (RE).

Whether recycling GPI-APs are delivered to the Golgi or the RE has been a point of debate, so the authors monitored the endocytic destinations of various GPI-APs - the folate receptor, decay-accelerating factor and GPI-green fluorescent protein (GFP) - in two cell types. They detected all endocytosed GPI-APs in the RE, but not in the Golgi, in both cases.

Although internalized GPI-APs colocalize with transferrin - an endocytosed marker of the RE - a fraction of these GPI-APs consistently remains segregated from transferrin and the Golgi. To find out what this means, Mayor and co-workers compared the early steps in GPI-AP endocytosis with markers of the clathrin-mediated endocytosis pathway, and found that immediately after internalization, a large fraction of GPI-APs is present in compartments that are distinct from transferrin-receptor-containing endosomes. The authors named these tubular-vesicular compartments 'GPI-AP-enriched early endosomal compartments' (GEECs).

Mayor and colleagues showed that GEECs do not colocalize with compartments marked by GFP-rab5 and GFP-rab4 — markers that colocalize with transferrin in sorting endosomes. This further indicates that GEECs are distinct from the classical clathrin-pit-derived sorting endosomes. The authors also found that GEECs constitute an important pinocytic pathway in the cells studied.

Internalization into GEECs specifically requires the GPI anchor, as the authors found that transmembrane isoforms of GPI-APs and a transferrin receptor lacking its cytoplasmic tail were excluded from GEECs. They also showed that GEEC uptake of both GPI-APs and the fluid phase is independent of dynamin II and caveolae - other markers of well-established routes of endocytosis (see page 311).

As the Rho family of GTPases are known to have a regulatory function in endocytic processes, Mayor and co-workers investigated their effect on GEEC formation. They found that inactivation of Cdc42 but not RhoA or Rac1 - inhibits the GEEC pathway, which reduces fluid-phase uptake and results in a redirection of GPI-APs to the clathrin-mediated endocytic pathway.

Endocytosis of GPI-APs seems to be important for their function and pathology - for example, the GPI-anchored prion protein is converted to its infectious form after internalization. The discovery by Mayor and colleagues of a new Cdc42-regulated, clathrin- and caveolae-independent, recycling route for these proteins is therefore likely to be of considerable biological and immunological importance.

(2) References and links

Rachel Smallridge

ORIGINAL RESEARCH PAPER Sabharaniak, S. et al. GPI-anchored proteins are delivered to

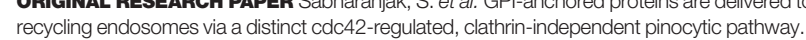
recycling endosomes via a

Dev. Cell 2, 411-423 (2002)
FURTHER READING Chatterjee, S. \& Mayor, S. The GPI-anchor and protein sorting. Cell Mol. Life Sci. 58, 1967-1987 (2001) 